\title{
Publisher Correction: Eternal blood vessels
}

\section{Jordan Hindson}

Nature Reviews Materials https://doi.org/10.1038/s41578-018-0015-x (2018)

Published online 26 April 2018

This article was originally published with an incorrect reference for the original article. The reference has been amended. Please see the correct reference below.

Qiu, Y. et al. Microvasculature-on-a-chip for the long-term study of endothelial barrier dysfunction and microvascular obstruction in disease. Nat. Biomed. Eng. https://doi.org/10.1038/ s41551-018-0224-z (2018)

https://doi.org/10.1038/s41578-018-0019-6 I Published online 02 May 2018 